\title{
Mechanisms and models of control of turbulent transfer in boundary layer
}

\author{
Kovalnogov Vladislav N. \\ Department of Heat-and-Power Engineering \\ Ulyanovsk State Technical University \\ Ulyanovsk, Russian Federation \\ kvn@ulstu.ru \\ Maleshin Igor A. \\ Department of Heat-and-Power Engineering \\ Ulyanovsk State Technical University \\ Ulyanovsk, Russian Federation \\ malyoshin.igor@mail.ru
}

\author{
Fedorov Ruslan V. \\ Department of Heat-and-Power Engineering \\ Ulyanovsk State Technical University \\ Ulyanovsk, Russian Federation \\ r.fedorov@ulstu.ru \\ Suranov Dmitry V. \\ Department of Heat-and-Power Engineering \\ Ulyanovsk State Technical University \\ Ulyanovsk, Russian Federation \\ suranoff@yandex.ru
}

\author{
Khakhaleva Larisa V. \\ Department of Heat-and-Power Engineering \\ Ulyanovsk State Technical University \\ Ulyanovsk, Russian Federation \\ larvall@mail.ru
}

\begin{abstract}
The work proposes the model of turbulent transfer in a boundary layer, which reflects the influence of periodic control actions on turbulent transfer factors. It provides the opportunity of reasonable turbulent transfer management. The list of actions, which influence turbulent transfer intensity, is revealed. The work describes the results obtained in researching the heat-transfer and the friction of a turbulent flow.
\end{abstract}

Keywords-boundary layer; turbulence; heat-and-mass transfer; mathematical modeling

\section{INTRODUCTION}

Control of turbulent transfer in a boundary layer allows influencing effectively the intensity of heat and mass transfer and resistance of friction of a moving stream of working media in elements of power devices, transport systems, etc. In the literature, a number of methods and devices for both heat transfer and resistance intensification, and their reduction is offered [1-10]. For example, imposing acoustic field or ultrasonic fluctuations of pressure on a moving stream; using channels with alternating narrowed and extending sites; using the punched surfaces with deaf damping cavities. Also the paper demonstrates the application of heat exchanging surfaces with cross gofers or elements of discrete roughness, on which the changing of a longitudinal gradient of pressure at length of a streamline surface, curvature of a surface, etc. is realized,

Search and practical realization of optimum methods of management by turbulent transfer restrains insufficient development of the theory of turbulence. It concerns a problem of the adequate account of various managing influences on intensity of turbulent transfer in a boundary layer, as well as the mathematical description of processes of interaction of managing elements and turbulent formations with various scales. For example, the interaction of surface roughness and perforations in a turbulent flow are considered as a whole. Development of corresponding theoretical generalizations on turbulent transfer in a near-the-wall boundary layer under managing influence allows proving a basic opportunity and limits of such management, establishing the list of human-aging influences and establishing their interrelation with characteristics of turbulent transfer in a boundary layer.

\section{INITIAL PRECONDITIONS AND CONCLUSIONS BY RESULTS OF EXPERIMENTAL RESEARCH OF LAWS OF TURBULENT TRANSFER IN A BOUNDARY LAYER UNDER INFLUENCES}

Within the framework of the accepted approach, the direct and indirect influences of managing effect on mean parameters of flow in a boundary layer, and, hence, on the intensity of heat transfer, friction, efficiency of a veil, are allocated. Thus, it means that the system of the differential equations of a turbulent boundary layer together with initial and boundary conditions correctly reflects only direct effect of various influences. Indirect effect of the mentioned influences is shown through the change of factors of turbulent transfer of heat and quantity of movement.

Effect of various influences on factors of turbulent transfer was studied experimentally with the use of the original automated thermo-anemometer complex [7]. With the use of 
this complex, the experimental research of laws of turbulent transfer in a boundary layer of stationary and dynamically non-stationary (pulsing) streams in channels of the different form [7] is executed. It is established by the researches that managing influences result in such features of turbulent transfer which essentially cannot be taken into account neither with the help of the classical "way of mixture" model of Prandtl nor with the help of the differential two-parametrical "k-eps" model of turbulence. When the kinetic energy of the turbulent flow is changed monotonically, it does not include the influence of a longitudinal gradient of pressure and the non-stationarity factor on the intensity of turbulent pulsations (and intensity of turbulent transfer) in a boundary layer. At the same time, for the use of more complex multiple-parameter models of turbulent transfer, there is no necessary volume of the empirical information.

Resulting influence of various influences on heat transfer and friction in a boundary layer within the framework of the accepted approach is analyzed on the basis of numerical integration of the system of the differential equations of a boundary layer (stationary and non-stationary). Simultaneously in the equations, the real characteristics of turbulent transfer, which were pawned on the basis of an experimental research of a turbulent structure of streams, are established. The adequacy of the results of the numerical study is confirmed by comparing the data with the known results of experimental studies.

\section{MATHEMATICAL MODEL OF BOUNDARY LAYER UNDER} ACTIONS

\section{A. System of equations}

The boundary layer equations system under actions is presented as:

$$
\begin{gathered}
\rho c_{p}\left(\frac{\partial T}{\partial \tau}+u \frac{\partial T}{\partial x}+v \frac{\partial T}{\partial y}\right)=\frac{1}{r^{n}} \frac{\partial}{\partial y}\left[r^{n}\left(\lambda+\lambda_{T}\right) \frac{\partial T}{\partial y}\right]+ \\
+\left(\mu+\mu_{T}\right)\left(\frac{\partial u}{\partial y}\right)^{2}+\frac{\partial p}{\partial \tau}+u \frac{\partial p}{\partial x}+q_{v} \\
\rho\left(\frac{\partial u}{\partial \tau}+u \frac{\partial u}{\partial x}+v \frac{\partial u}{\partial y}\right)=\frac{1}{r^{n}} \frac{\partial}{\partial y}\left[r^{n}\left(\mu+\mu_{T}\right) \frac{\partial u}{\partial y}\right]- \\
-\frac{\partial p}{\partial x}+s_{v} ; \\
\frac{\partial \rho}{\partial \tau}+\frac{1}{r^{n}}\left[\frac{\partial}{\partial x}\left(\rho u r^{n}\right)+\frac{\partial}{\partial y}\left(\rho v r^{n}\right)\right]=0 \\
\rho=\rho(T) ; \mu=\mu(T) ; \lambda=\lambda(T) ; c_{p}=c_{p}(T) .
\end{gathered}
$$

Here $x, y, \tau$ are, accordingly, longitudinal (along the surface), cross (on a normal line to a surface) coordinate and time. $n$ is the parameter: 1 - for axially symmetric, and 0 for a flat boundary layer. $u, v, t, p$ are projections of a vector of a velocity of a stream on coordinate axes $x, y$, its temperature and pressure respectively. $\rho, \mu, \lambda, c_{p}$ are density, dynamic coefficients of viscosity, thermal conductivity coefficient and specific isobaric thermal capacity, accordingly. $\mu_{t}, \lambda_{t}$ are coefficients of turbulent transposition of a momentum and heat; $r$ is a radius; $R$ is a gas constant. $s_{v}, q_{v}$ are terms describing intensity of interior sources of momentum and heat accordingly $[8,9,10]$.

The dependences (4) of thermal properties on temperature are given.

\section{B. Initial and boundary conditions}

Initial and boundary conditions are:

$$
\begin{gathered}
\tau=0: u=u_{0}(x, y) ; T=T_{0}(x, y) \\
\tau>0: x=0 ; u=u(y, \tau) ; T=T(y, \tau) ; \\
y=0 ; u=0 ; v=v_{w}(x, \tau) ; T=T_{w}(x, \tau) ; \\
y=0 ; u=0 ; v=v_{w}(x, \tau) ; \partial T / \partial y=-q_{w} / \lambda ;
\end{gathered}
$$

$$
y=\infty ; \partial u / \partial y=0 ; \partial T / \partial y=0
$$

Here $q$ is heat flux density; index 0 refers to parameters at initial instant; index $w$ refers to parameters on a wall surface.

Pressure $p$, as well as velocity $u_{\infty}$ and temperature $T_{\infty}$ outside a boundary layer are defined as a result of calculation of non-viscous ideal fluid in a flow core. Therefore in a problem of calculation of a boundary layer, these parameters are considered as known for each cross-section $x$ at each instant $\tau$.

The boundary conditions for the equation of energy (1) are given in two forms: (7a) and (7b). These conditions are used during calculation of a boundary layer on cooled (or heated) surface, as the result of which the coefficients of the convective heat transfer can be defined. Condition (7a) is used also during calculation of a boundary layer on the adiabatic surface (in this case, adiabatic wall temperature and efficiency of a gas (or thermal) veil are defined).

\section{Model of turbulent transfer}

According to the classical Prandtl model of the merging path, the coefficient of turbulent transfer of momentum $\mu_{T}$ is determined by the relation: 


$$
\mu_{T}=\rho l^{2} \partial u / \partial y
$$

where $l$ is the merging path length near the wall, which is determined by the expression:

$$
l=x y\left\{1-\exp \left[-\rho v_{*} y /(26 \mu)\right]\right\},
$$

where $v_{*}$ is dynamic velocity in a considered point; $x$ is the coefficient describing intensity of turbulent transfer of a momentum.

The coefficient of turbulent transfer of heat $\lambda_{T}$ is connected with coefficient $\mu_{T}$ by the relation:

$$
\lambda_{T}=\mu_{T} c_{p} / \operatorname{Pr}_{T}
$$

where $\operatorname{Pr}_{T}$ is turbulent Prandtl number.

The classical merging path model is developed by Prandtl for a stationary boundary layer in homogeneous non-gradient flow. Therefore, it does not allow one to predict immediate influence on coefficients of turbulent transfer of exterior perturbing actions and various types of non-stationarity. In frames of this model, coefficient $æ$ and number $\operatorname{Pr}_{T}$ are considered as constants $\left(\not 2=0.4 ; \operatorname{Pr}_{T}=0.9\right)$.

Undertaken experimental research of the turbulent structure of stationary and non-stationary boundary layers, subjected to various actions [7], has allowed one to modify the classical Prandtl model to extend it by non-stationary and stationary flows with various actions. It is established that in a non-stationary boundary layer with influences, coefficient $æ$ and number $\operatorname{Pr}_{T}$ do not remain constants. They vary in time and along a circulated surface. Under such conditions, the author has obtained and experimentally confirmed the following expressions for turbulent Prandtl number $\operatorname{Pr}_{T}$ and coefficient $æ$ :

$$
\begin{gathered}
\operatorname{Pr}_{T}=\frac{0.9}{1+10^{7} \frac{\left(\partial T_{w} / \partial \tau\right)}{u_{0 \infty}(\partial T / \partial y)_{y=0}}} \\
x=0.4 \frac{\sqrt{1-\frac{4.9}{\rho_{\infty} u_{\infty}(\partial u / \partial y)_{y=0}}\left[\frac{\partial p_{\infty} / \partial \tau}{u_{0 \infty}}+\left(\frac{\partial p}{\partial y}\right)_{\max }\right]}}{1-21.4 \frac{\partial p_{\infty} / \partial x}{\rho_{\infty} u_{0 \infty}(\partial u / \partial y)_{y=0}}}
\end{gathered}
$$

Here index $\infty$ refers to the parameters on exterior boundary of a boundary layer in considered cross-section at the considered instant; $0 \infty$ is the same in the initial cross-section. Expression (13) for coefficient $æ$ is simplified in some cases for a stationary boundary layer. For flows with a longitudinal gradient of pressure, this expression will be transformed to a kind:

$$
x=0.4\left(1-21.4 \frac{d p / d x}{\rho_{\infty} u_{0 \infty}(\partial u / \partial y)_{y=0}}\right)^{-1}
$$

In a boundary layer on a curvilinear surface:

$$
æ=0.4 \sqrt{1-\frac{4.9 u_{\infty}}{R(\partial u / \partial y)_{y=0}}},
$$

where $R_{n}$ is radius of curvature of a surface $\left(R_{n}>0\right.$ for a convex surface, $R_{n}<0$ for a concave surface).

In a boundary layer on a rotating surface:

$$
x=0.4 \sqrt{1 \pm \frac{4.9 \omega}{(\partial u / \partial y)_{y=0}}},
$$

where $\omega$ is angular speed of rotation.

The turbulent pulsations in any point of a boundary layer react to actions with some delay. Therefore, using expressions (12) and (13) for the analysis of a boundary layer with periodic actions, this delay should be taken into account. The relaxation time $\tau_{*}$, representing time of turbulent pulsations energy diffusion from a zone of their origin (viscous underlayer) up to a considered point inside a boundary layer. Time $\tau_{*}$ can be expressed by the relation:

$$
\tau_{*}=l / v_{*_{w}}
$$

where $v_{*_{w}}$ is dynamic velocity on a circulated surface.

Relevant to time $\tau_{*}$ relaxation length $x_{*}$ is defined by the expression:

$$
x_{*}=u \tau_{*} .
$$

The definition of values of magnitudes $æ$ and $\operatorname{Pr}_{T}$ under considered conditions is realized when using expressions (12) and (13), but instantaneous (relevant to a considered instant) and local (relevant to considered cross-section) values of influential parameters in their right-hand sides are substituted by time $\tau_{*}$ averaged or length $x_{*}$ averaged ones using the expressions:

$$
\bar{\varphi}=\frac{1}{\tau_{*}} \int_{-\tau-\tau_{*}}^{\tau} \varphi d \tau ; \bar{\psi}=\frac{1}{x_{*}} \int_{x-x_{*}}^{x} \psi d x
$$


Here $\varphi$ is any value of forth parameters $\partial p_{\infty} / \partial \tau, \partial u_{\infty} / \partial \tau, \partial T_{w} / \partial \tau,(\partial p / \partial y)_{\max }$ in a considered instant; $\bar{\varphi}$ is an averaged value, which is substituted in expressions (12) and (13); $\psi$ is any local value of parameters $\partial p_{\infty} / \partial x, \partial u_{\infty} / \partial x,(\partial p / \partial y)_{\max } ; \bar{\psi}$ is an averaged value, which is substituted in expressions (12) and (13).

\section{Interior sources of disperse flows of heat and momentum}

Magnitudes $q_{v}$ and $s_{v}$ in a disperse boundary layer can be defined using relations:

$$
\begin{gathered}
q_{v}=\frac{6}{\rho_{B}} \sum_{i=1}^{m} \frac{\alpha_{s i} \rho_{s i}}{d_{s i}}\left(T_{s i}-T\right) ; \\
s_{v}=\frac{3}{4} \frac{\rho}{\rho_{B}} \sum_{i=1}^{m} \frac{\rho_{s i} c_{f s i}}{d_{s i}}\left|u_{s i}-u\right|\left(u_{s i}-u\right) .
\end{gathered}
$$

Here $\alpha_{s}, c_{f s}$ are a coefficient of a convective heat transfer and a resistance coefficient of particles; $\rho_{s}$ is density of a phase condensed (mass of particles condensed per unit of medium volume); $\rho_{B}$ is density of substance of particles; $d_{s}$ is a diameter of a particle; $m$ is the amount of the chosen fractions of particles distinguished by the size.

To define density $\rho_{s}$ of a condensed phase, velocity $u_{s}$ of a particle and its temperature $T_{s}$, the calculation of trajectories and parameters of particles for the several chosen fractions for the beforehand calculated field of fluxion of the carrier medium is carried out (generally, the calculation can be carried out by a method of successive approximations). It is expedient to include a representative fraction with massaveraged particles dimension $d_{s m}$ in the number of these fractions. Temperature $T_{s m}$ and velocity $u_{s m}$ of particles of a representative fraction are used in addition to other parameters for calculation of complexes $A$ and $B$ :

$$
A=q_{v} /\left(T_{s m}-T\right) ; \quad B=s_{v} /\left(u_{s m}-u\right) .
$$

The calculations show that complexes $A$ and $B$ vary (both by the width of a boundary layer, and in different iterations during improvement of a field of fluxion of a carrier medium), which is much more feeble than magnitudes $q_{v}$ and $s_{v}$. Therefore, it is expedient to average complexes $A$ and $B$ retrieved in the previous approach through the analyzable section of a boundary layer and to use these average values for definition of local parameters $q_{v}$ and $s_{v}$ by expressions:

$$
q_{v}=\bar{A}\left(T_{s m}-T\right) ; \quad s_{v}=\bar{B}\left(u_{s m}-u\right)
$$

where $\bar{A}, \bar{B}$ are average (through the boundary layer width) values of complexes $A$ and $B$.

It is recommended to approximate the profiles of temperature $T_{s m}$ and velocity $u_{s m}$ of particles of the representative fraction in sections of a boundary layer by dependences:

$$
\begin{aligned}
& u_{s m}=u_{s m \infty}\left[\varphi_{u}+\left(1-\varphi_{u}\right) \frac{u}{u_{\infty}}\right] ; \\
& T_{s m}=T_{s m \infty}\left[\varphi_{T}+\left(1-\varphi_{T}\right) \frac{T}{T_{\infty}}\right] .
\end{aligned}
$$

Coefficients $\varphi_{u}$ and $\varphi_{T}$, which maintain the stationary values in analyzable section of a boundary layer, can range from 0 up to 1 and their values depend in basic on width of a boundary layer, dimensions of particles and velocity of their cross travel. The more velocity of cross travel of a particle or the bigger its diameter or the less the width of a boundary layer, the closer to 1 the value of these coefficients. In those areas, where the velocity of directional cross travel of particles is equal 0 (inertial dropout of particles on a wall misses), the coefficients $\varphi_{u}$ and $\varphi_{T}$ accept values of close to 0 .

After definition of numerical values of coefficients $\bar{A}, \bar{B}$, $\varphi_{u}$ and $\varphi_{T}$, there is an opportunity to calculate interior sources $q_{v}$ and $s_{v}$ during integration of the equations of a boundary layer with the help of expressions (21) and (22) instead of autonomous calculation by a method of successive approximations.

\section{SOME RESULTS OF RESEARCH}

\section{A. Convective heat transfer of a turbulent non-stationary stream in a nozzle}

The calculations are carried out for a Laval nozzle with reference to conditions of sudden starting of a hot flow through a cold working site. The time history of flow parameters (velocity, temperature) on the exterior border of a boundary layer and temperature of the surface of the wall in three sections of a nozzle is shown in fig. 1. 

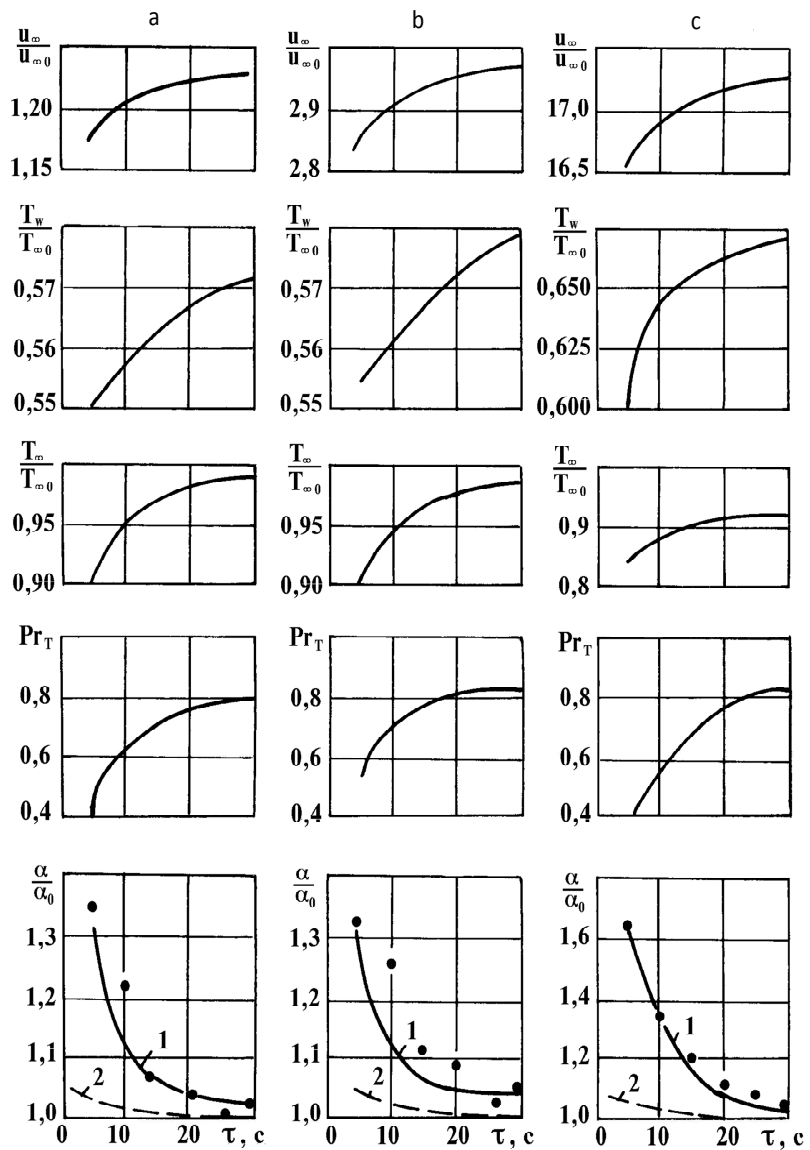

Fig. 1. The performances of a non-stationary boundary layer in different sections of a nozzle ( $\mathrm{a}$ - input section; $\mathrm{b}$ - convergent site; $\mathrm{c}$ - critical section): 1 - calculation by the model offered taking unto account the change of $\operatorname{Pr}_{T} ; 2$ calculation at constant value $\operatorname{Pr}_{T}=0.9 ; \bullet-$ experimental data obtained by the authors

In the same figure the values, obtained by the calculations, of a turbulent Prandtl number and coefficient $\alpha$. Here $\alpha_{0}$ is steady-state value of coefficient of a convective heat transfer. As it is visible, the calculation on model offered adequately reflects influence of the non-stationarity and longitudinal lapse rate of pressure in a Laval nozzle. It is possible to mark also, that the basic contribution in intensification of a convective heat transfer (reaching $60 \%$ in conditions viewed) renders the change of a turbulent Prandtle number. The calculations have shown also, that coefficient $\mathscr{X}$ in sections of a boundary layer of a nozzle appears much less than value 0,4 (the effects of a laminarization), that leads to the $60 \%$ drop of intensity of a convective heat near the critical section.

\section{B. Convective heat transfer and efficiency of a veil at a motion of a stationary disperse flow in a nozzle}

The results of numerical examination of a convective heat transfer of a flow of a gas mixture in a nozzle and efficiency of its interior cooling $\Theta$ are shown in fig. 2 and 3. The results given correspond to number $\operatorname{Re}=0.93 \cdot 10^{5}$ (number $\operatorname{Re}$ is counted from flow parameters and a tract diameter on a nozzle inlet) and average concentration of particles in the flow $\widetilde{\beta}=0.825$. As it is visible from fig. 2 , the calculation by the model offered adequately reflects the character of change of the relative coefficient of a convective heat transfer on the length of the channel. Quantitative mismatch experimental and calculation datasets in sections $\bar{x}>0.4$ are interconnected mainly with gradual particle accumulation in the near-the-wall zone, which action on the turbulent transfer in a boundary layer of the carrier medium was not taken into account during calculations. As it is visible from fig. 2, 3 that the presence of directional inertial particle fluxes on the surface leads to the significant (up to 2.5 times) intensification of the convective heat transfer and a more than 2-time reduction of the efficiency of interior cooling. The drop of veil efficiency is explained by the fact that the particles condensed promote additional heating of the boundary layer during their inertial travel from a high-temperature flow core to a low-temperature near-the-wall stratum.

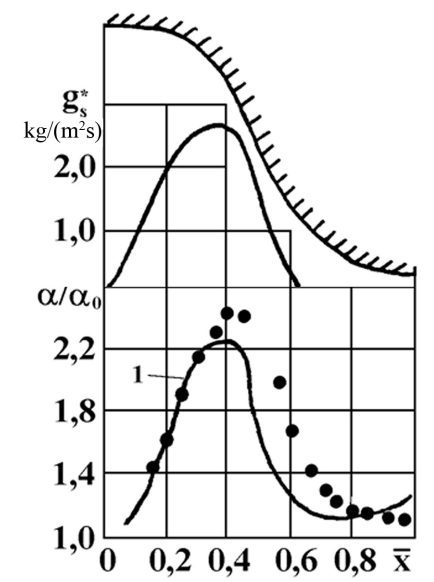

Fig. 2. Allocation (through the length of a nozzle) of density of mass flux $g_{s}^{*}$ of particles condensed on a wall and a relative coefficient of a convective heat transfer: 1 - values calculated; $\bullet$ - experimental data

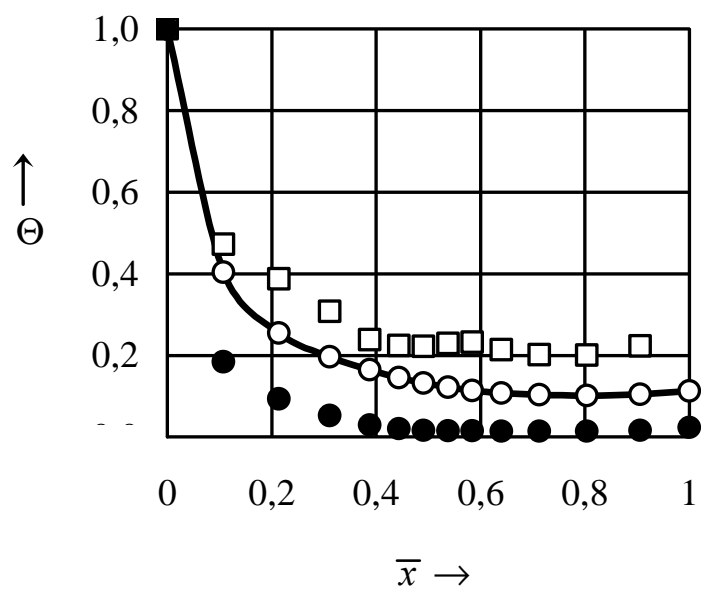

Fig. 3. Results of calculation of efficiency of interior cooling of a nozzle: $\mathrm{O}$ - homogeneous flow; $\bullet$ - disperse flow in the presence of inertial dropout of particles on a wall; $\boldsymbol{\square}$ - the same in the absence of inertial dropout 
In the subsequent series of calculations, the situation of missing the inertial dropout of condensed particles on the wall was simulated. It happens with quite small sizes of particles in the flow or with the sufficiently large sizes of a tract. In the absence of directional cross travel of particles in a boundary layer, their influence on a convective heat transfer in conditions considered appeared to be minor. At the same time, the efficiency of a disperse veil in the flow of a gas mixture essentially increases in comparison with a homogeneous flow (fig. 3). It is explained by the fact that in this case, particles move along streamlines of gas, and in the near-the-wall zone they move from sites with low temperature to sites with high temperature, promoting additional cooling of gas in a veil.

\section{Convective heat transfer and friction of a plate during simple harmonic pressure oscillations of a homogeneous flow}

The numerical integration of the system of the differential equations of a boundary layer was executed with the definition of average (for the first period of oscillations of parameter explored) values of density of heat flow $q_{w}$ and stress of friction $\tau_{w}$ on a streamline surface for all interesting sections. The initial allocation of parameters in a boundary layer is assumed to be relevant to stationary conditions of fluxion. The pressure oscillations for all sections of a boundary layer were assumed to be co-phased, having identical amplitude and frequency. Some results of the research obtained for a plate, which is flowered by an incompressible fluid with stationary properties, are given in fig. 4. Here $q_{w 0}, \tau_{w 0}$ are steady-state values of density of the heat flux on the wall and friction stresses on its surface; $f_{0}=f \mu_{0} /\left(\rho u_{0}^{2}\right)$ is dimensionless oscillation frequency of pressure; $f$ is dimensional frequency; $u_{0}, \mu_{0}$ are scale values of the velocity and dynamic coefficient of viscosity of the flow; $\mathrm{Eu}_{p}=p /\left(\rho u_{0}^{2}\right)$ - Euler number by pressure.

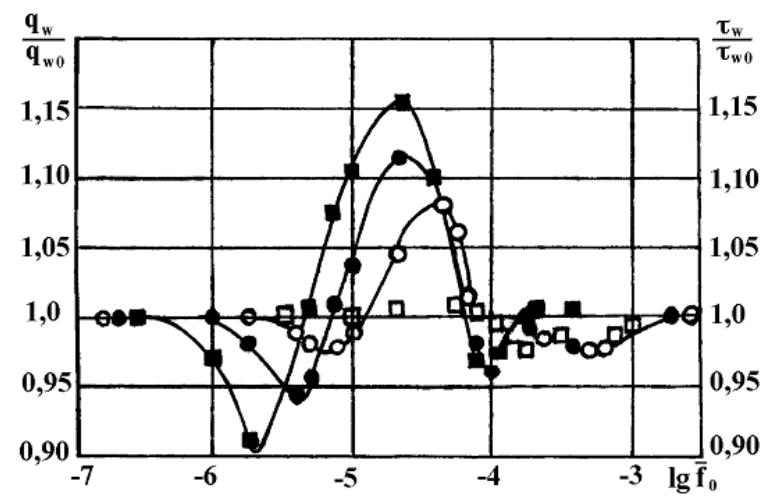

Fig. 4. Influence of frequency of simple harmonic pressure oscillations of the flow on the average (for one period) value of density of a heat flux and friction stress on the surface of a plate: $\mathbf{\square}-\mathrm{Eu}_{p}=400 ;-200 ; \bigcirc-100$; $\square-10$
Fig. 4 shows that the boundary layer reacts to periodic oscillations of pressure only in a particular frequency band. Dependence of dimensionless frequency of pressure oscillations on magnitudes $q_{w}$ and $\tau_{w}$ is non-monotone. On different sites of the frequency band, both impairment and enhancement of a convective heat transfer and resistance are possible. In accordance with magnification of number $\mathrm{Eu}_{p}$, the degree of influence of simple harmonic pressure oscillations on the convective heat transfer and resistance of a turbulent flow is incremented, and extremes of functions $q_{w}$ and $\tau_{w}$ displace to smaller frequencies. With diminution, frequency values relevant to a minimum of functions are lower than $q_{w}$ and $\tau_{w}$. There is an impairment of influence of pressure oscillations, which is interlinked with transition to a quasistationary mode of turbulent transfer. The impairment of influence of pressure oscillations also happens with magnification of frequency above the value relevant to a maximum of functions $q_{w}$ and $\tau_{w}$. It is stipulated by transition to a fast-oscillating mode of the "frozen" turbulence.

\section{Acknowledgment}

The reported study was funded by RFBR according to research project No. 16-31-60114 mol_a_dk.

\section{References}

[1] V.N. Kovalnogov, R.V. Fedorov, L.V. Khakhaleva, A.V. Chukalin, A.A. Bondarenko, and E.N. Kovrizhnykh, "The mechanism and theoretical basis of the management of intensity of the heat transfer control through periodic influences on the turbulent boundary layer", AIP Conference Proceedings, vol. 1863(560016), pp 1-5, 2017.

[2] V.F. Potemkin, G.A. Dreitser, "Way of management of a turbulent boundary layer", USSR Patent, no. 909384, 1982.

[3] A.L. Ionov, L.P. Ionov, A.A. Rozhnev, I.G. Novikova, "Device for decrease of friction in pipelines and hoses", USSR Patent, no. 500383, 1976.

[4] V.V. Babenko, L.F. Kozlov, V.I. Korobov, "Adjustable damping a covering”, USSR Patent, no. 597866, 1978.

[5] E.S. Vinogradov, "Surface, streamline a liquid or gas", USSR Patent no. 1086246, 1984.

[6] R.L. Panton, K.P. Flinn, D.G. Bogard, "Control of turbulence through a row of Helmholtz resonators", AIAA Pap., vol. 436, pp. 1-7,1987.

[7] N.N. Kovalnogov, Boundary layer in flows under intensive actions. Ulyanovsk: UlSTU, 1996, 246 p.

[8] V.N. Kovalnogov, and R.V. Fedorov, "Numerical analysis of the efficiency of film cooling of surface streamlined by supersonic disperse flow", AIP Conference Proceedings, vol. 1648(850031), pp 1-5, 2015.

[9] A.G. Laptev, "Mathematical model for mixing liquids with a disperse phase in laminar and turbulent regimes in packed-bed flow mixers", Theoretical foundations of chemical engineering, vol. 49(1), pp. 21-29, 2015.

[10] V.K. Shchukin, "Heat transfer of gas suspension in nozzles", Izvestiya Vysshikh Uchebnykh Zavedenii Aviatsionaya Tekhnika, vol. 3, pp. 61661979. 